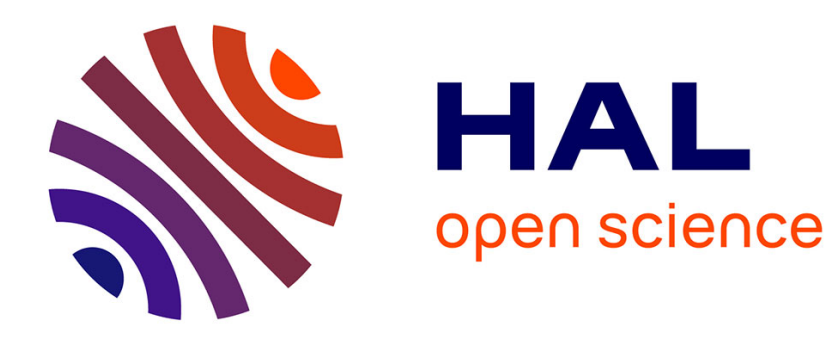

\title{
Neutron reflectivity studies of ordered copolymer films
}

\author{
A. Mayes, T. Russell
}

\section{To cite this version:}

A. Mayes, T. Russell. Neutron reflectivity studies of ordered copolymer films. Journal de Physique IV Proceedings, 1993, 03 (C8), pp.C8-41-C8-47. 10.1051/jp4:1993809 . jpa-00252228

\section{HAL Id: jpa-00252228 https://hal.science/jpa-00252228}

Submitted on 1 Jan 1993

HAL is a multi-disciplinary open access archive for the deposit and dissemination of scientific research documents, whether they are published or not. The documents may come from teaching and research institutions in France or abroad, or from public or private research centers.
L'archive ouverte pluridisciplinaire HAL, est destinée au dépôt et à la diffusion de documents scientifiques de niveau recherche, publiés ou non, émanant des établissements d'enseignement et de recherche français ou étrangers, des laboratoires publics ou privés. 


\title{
Neutron reflectivity studies of ordered copolymer films
}

\author{
A.M. MAYES and T.P. RUSSELL*
}

Department of Materials Science and Engineering, Massachusetts Institute of Technology, 77 Massachusetts Ave., Cambridge, MA 02154, U.S.A.

${ }^{*}$ I.B.M. Research Division, Almaden Research Center, 650 Hary Rd., San Jose, CA 95120, U.S.A.

\begin{abstract}
In a thin film geometry symmetric block copolymers selfassemble to form well-oriented multilayered structures, ideally suited for study by reflectivity techniques. Neutron reflectivity was performed on selectively deuterated poly (styrene-b-methyl methacrylate) copolymers to determine quantitatively the spatial distribution of monomer segments within the lamellar microdomains. The reflectivity curves are shown to be highly sensitive to gradients in the scattering length density normal to the film surface. The scattering length density profiles which best model the experimental data yield segment density profiles in ordered copolymers with unprecedented detail. The approach is extended to analyze chain distributions in multicomponent systems, including mixtures of symmetric copolymers with different molecular weights and mixtures of copolymers and homopolymers.
\end{abstract}


JOURNAL DE PHYSIQUE IV

\section{Introduction}

For nearly two decades polymer scientists have been intrigued by the morphologies formed through the local segregation of incompatible blocks in a block copolymer melt. The observed morphologies range from ordered arrays of spheres, cylinders and lamellae to continuous network structures, depending on the relative block fractions and the copolymer molecular weight. Until recently, characterization of these microdomain morphologies was limited primarily to the evaluation of domain size and spacing via small-angle $x$-ray and neutron scattering and transmission electron microscopy. Such information afforded only a qualitative description of block configurations within the ordered domains. In the past few years, however, the technique of neutron reflectivity has opened a gateway to quantitative analysis of polymer configurations in restricted geometries. Thus, segment density profiles in ordered copolymer systems can now be evaluated systematically.

In thin films of block copolymers a difference in surface energies of the two components or the chemical affinity of one component for the underlying substrate typically leads to a strong orientational preference of the domains. For symetric block copolymers (copolymers with equal fractions of the two components) alternating lameliar domains form parallel to the plane of the film $[1,2]$. Such films provide excellent model systems for investigation by reflectivity. Using isotopic substitution of deuterium for hydrogen to provide contrast, the distribution of copolymer blocks or portions of blocks can be determined from neutron reflectivity profiles. Chain distributions in ordered multicomponent systems containing block copolymers can similarly be analyzed by selective deuteration of the blend components.

This paper summarizes recent efforts to characterize chain distributions in ordered thin films containing symetric poly(styreneb-methyl methacrylate) block copolymers [3-5]. On silicon substrates, these copolymers organize such that PMMA segregates preferentially to the substrate and PS, the lower surface energy component, segregates to the free surface [1]. Copolymers synthesized with selectively deuterated chain ends or junction points have been used to gain detailed pictures of the block configurations within pure copolymer films [3]. Mixtures of symmetric PS-b-PMMA copolymers with different molecular weights have also been studied, in order to characterize the distribution of long and short blocks within the lamellar domains [4]. Finally, the spatial distribution of PMMA or PS homopolymers blended with PS-b-PMMA copolymers has been examined as a function of homopolymer molecular weight [5].

\section{Experimental}

Poly (styrene-b-methyl methacrylate) block copolymers were purchased from Polymer Laboratories or synthesized by Dr. S. Smith of Proctor \& Gamble, following procedures detailed elsewhere [3]. Soxhlet extraction with cyclohexane removed any PS homopolymer impurities present in the copolymers as by-products of the syntheses. Polystyrene and poly (methyl methacrylate) homopolymers were purchased from Polymer Laboratories, and used as received. The copolymers were roughly symmetric in composition. All the materials were anionically synthesized, with polydispersities ranging from 1.04 and 1.13 [3-5].

Polished silicon substrates $10 \mathrm{~cm}$ in diameter were purchased from semiconductor Processing Co. Prior to use, substrates were placed in Chromerge overnight, rinsed thoroughly in deionized water and exposed 
to isopropanol vapor. This cleaning procedure leaves an oxide layer on the silicon surface. Thin films of the copolymers and blends were prepared by spin casting from $\sim 3$ wto solution in toluene onto the substrates to give films 1000-2000 $\AA$ thick. To achieve orientation, films were annealed at $170^{\circ} \mathrm{C}$ under vacuum for several days.

Neutron reflectivity measurements were performed on the BT-7 instrument at the National Institute of Standards and Technology in Gaithersburg, Maryland. The experimental setup and sample alignment procedure are described in detail elsewhere [1]. Reflectivity profiles were typically measured out to $.07 \AA^{-1}$ in neutron momentum, $\mathrm{k}_{\mathrm{z}, 0}=2 \pi / \lambda \sin \theta$, where $\lambda=2.37 \AA$.

To fit the reflectivity curves, model scattering length density $(b / V)$ profiles were generated by dividing the ordered copolymer films into many layers and calculating the $b / V$ value for each layer from an assumed form for the concentration profile within the copolymer domains [3-5]. The theoretical reflectivity was then derived from the model $b / V$ profile and evaluated against the experimental data. Model parameters were iteratively modified to achieve a best fit to the data. In the systems studied, the presence of three segment types (one deuterated and two hydrogenated monomers) complicates the transformation of the $b / V$ profiles into volume fraction profiles. For this a second experiment is required in which all monomer segments confined to the PS or PMMA domains are isotopically matched, and one block is fully deuterated while the other is unlabeled. This control experiment allows an independent measure of the average PS/PMMA

interfacial width, which one needs to deconvolute the three-component $b / V$ profiles. Knowing the measured interfacial widths and the volume fractions of the input materials, the $b / V$ values for each model layer can be converted into three weighted contributions [5].

\section{Results and Discussion}

Selectively labeled copolymers

One method to study block configurations in ordered copolymer domains is to selectively label sites along the block and evaluate their spatial distribution. In an earlier study, Matsushida et al. performed small-angle neutron scattering on symmetric poly(styrene-bvinyl pyridine) block copolymers selectively deuterated at the chain ends or junction points [6]. In explaining the sANS data, the authors proposed model concentration profiles in which the fraction of deuterated segments was localized entirely at the domain center (endlabeled) or domain edges (junction-labeled). While some degree of localization within the microdomains could be expected, such extreme models are probably unrealistic unless the chains are very highly stretched normal to the lamallae. This study thus shows the limitations of SANS in yielding such information quantitatively.

By comparison, neutron reflectivity demonstrates a high degree of sensitivity to label distributions in thin films of selectively deuterated copolymers [3]. Figure 1 shows the reflectivity data (open circles) from a center-labeled 100K mol. wt. PS-b-dPS-b-PMMA copolymer with 2.7 vol\% labeling. The scattering length density profile given in the inset corresponds to the best fit to the data (solid line). Although the fraction of deuterated material in the system is quite small (roughly 25 polystyrene segments) the contrast arising from the localization of these units to the PS/PMMA interface gives rise to strong Bragg reflections.

To convert the $b / V$ profille to volume fractions an interfacial width of $50 \AA$ was assumed, based on earlier results from dPS-b-PMMA and PS-b-dPMMA copolymers of comparable molecular weight [1]. The 


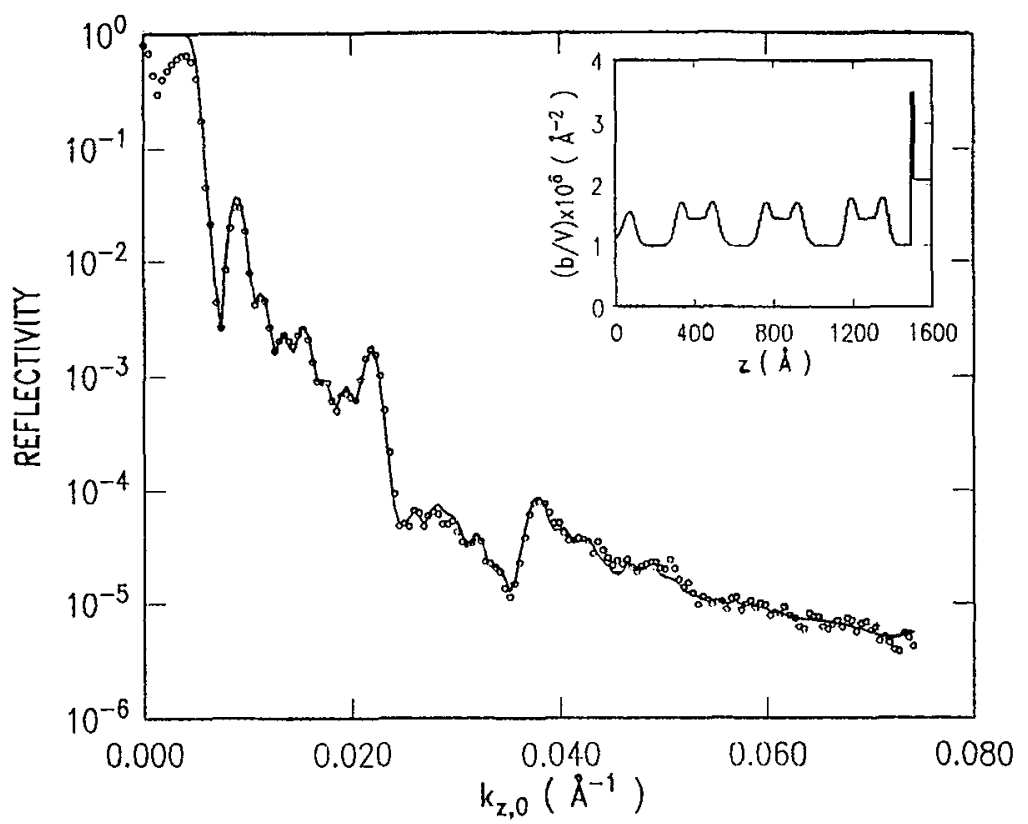

Figure 1. Reflectivity data $(0)$ and best fit (-) for a centerlabeled PS-b-PMMA copolymer.

concentration profile in a representative domain is shown in Figure 2a. The center dPS segments are essentially localized to the interface between the PS and PMMA domains, but asymmetrically distributed toward the PS side of the interface. This follows from the fact that the labeled PS has a finite chain length, and should thus reside preferentially in the PS domains.
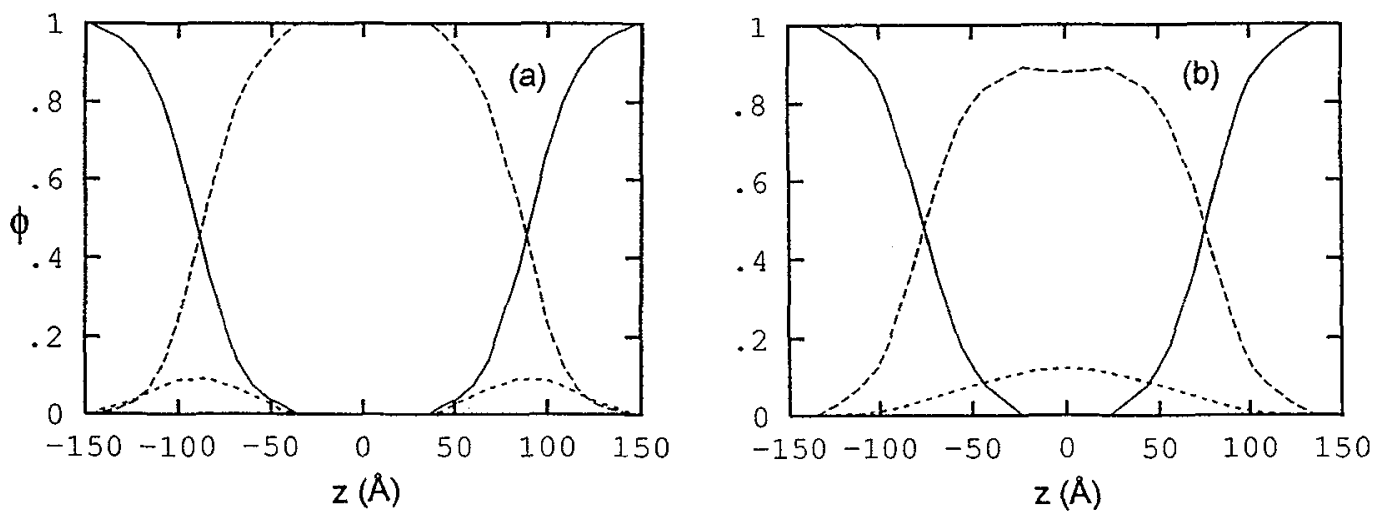

Figure 2. Distribution of junction points (a) and chain ends (b) in two ordered PS-b-PMMA copolymers. 
Although the junction points are fairly well localized, experiments on an end-labeled 70K PS-b-PMMA-dPMMA copolymer suggest that the block copolymer chain ends are much more evenly distributed within the copolymer domains. Figure $2 \mathrm{~b}$ shows the experimentally determined volume fraction profile for a representative domain of this copolymer, which contains 5.0 vol\% labeling. Only a slight centralization of the chain ends to the domain center is observed.

Copolymex-copolymer mixtures

In a small-angle scattering study on binary mixtures of copolymers with different molecular weights, Hadziioannou and skoulios found a linear variation in the domain spacing with concentration of the parent copolymers [7]. This result suggests that within the domains of such a copolymer mixture the long and short blocks are uniformly distributed. To investigate this possibility, symmetric 100K PS-b-PMMA copolymers were blended with minor fractions $(\leq 25$ vol:) of $300 \mathrm{~K}$ or $30 \mathrm{~K}$ PS-b-PMMA copolymers and analyzed in a thin film geometry by neutron reflectivity [4]. Figure 3 shows the reflectivity profile of a 90:10 mixture of 110K PS-b-PMMA and 300K dPS-b-PMMA. Although the fraction of high molecular weight material is moderate, the distribution of the $50 \mathrm{~K}$ and $150 \mathrm{~K}$ polystyrene blocks is still observed to be nonuniform across the PS domains, as indicated from the $b / V$ profile inset.

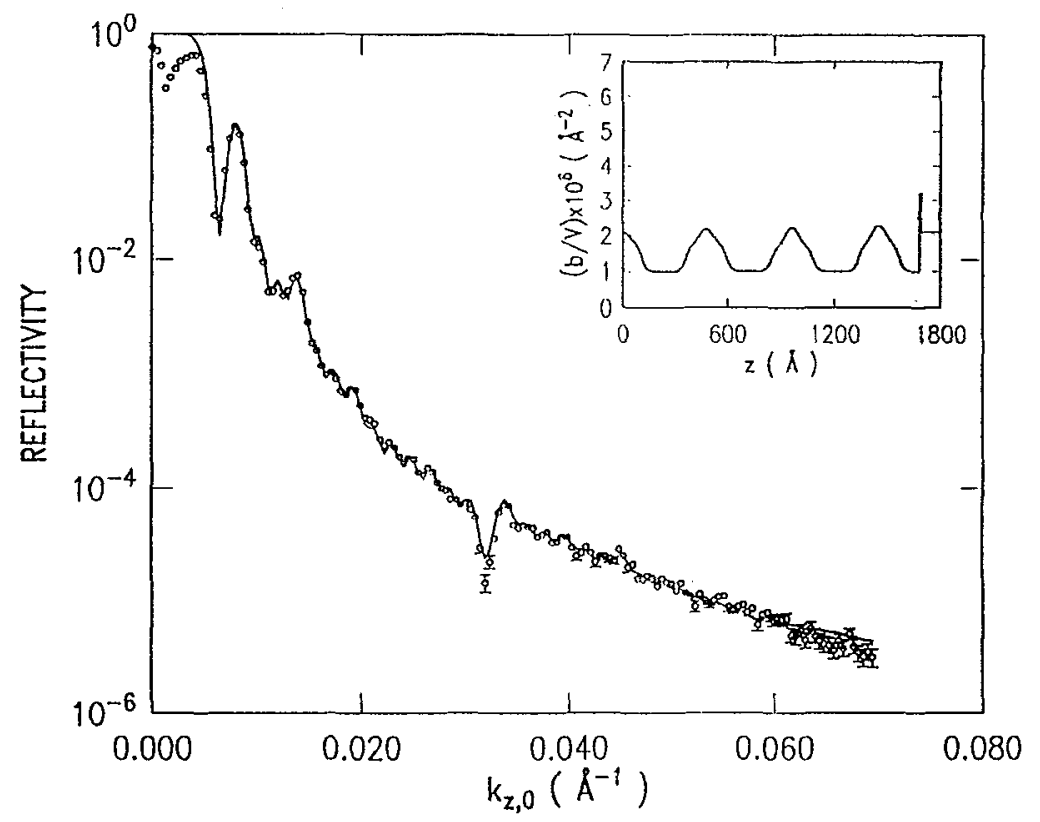

Figure 3. Reflectivity data (0) and best fit (-) for a 90:10 blend of $110 \mathrm{~K}$ PS-b-PMMA and $300 \mathrm{~K}$ dPS-b-PMMA.

In a control experiment, the average PS/PMMA interfacial width was determined for a 90:10 mixture of 120K dPMMA-b-PS with 300K dPMMA$b-P S$ to be $48 \AA$. The concentration profile deduced using this result is shown in Figure $4 \mathrm{a}$. The high molecular weight PS block is clearly in excess in the center of the PS domain, whereas the shorter blocks are preferentially located at the interface. 
Similar results are obtained when a $30 \mathrm{~K}$ aPS-b-PMMA copolymer is substituted for the $300 \mathrm{~K}$ copolymer. The concentration profile for this blend is shown in Figure $4 \mathrm{~b}$. For this system the interfacial width was found in a control experiment to expand to $65 \AA$ from the presence of the low molecular weight material. Nearly half of the short block segments rest within the interfacial region. Moreover, the short blocks do not appear to reach the center of the PS domains.
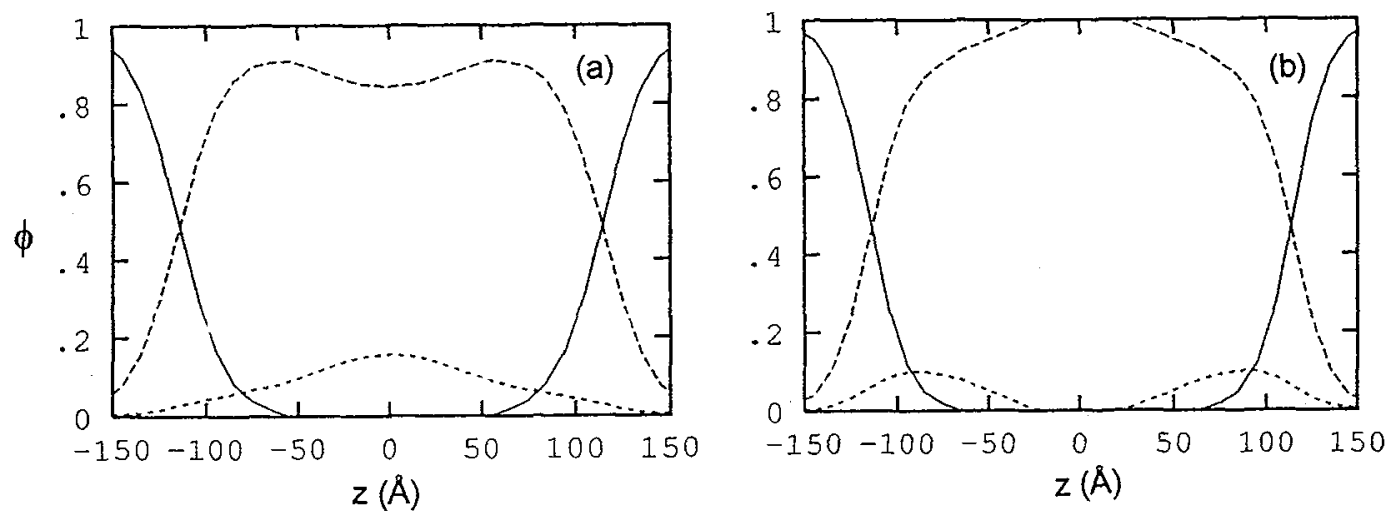

Figure 4. Distribution of long (a) and short (b) copolymer blocks in binary mixtures of symmetric PS-b-PMMA copolymers.

\section{Copolymer-homopolymer blends}

From SAXS and TEM studies conducted by two groups on ordered blends of $A-b-B$ copolymer with $A$ homopolymer, qualitatively different pictures of the homopolymer distributions were proposed $[8,9]$. To address this issue more quantitatively, neutron reflectivity was performed on thin film blends of PS or PMMA homopolymers with PS-bPMMA copolymers [5]. Figure 5a shows the concentration profile in a representative domain from a 90:10 blend of 90K PS-b-PMMA and 60K deuterated PMMA. The profile indicates a high degree of localization of the homopolymer chains to the center of the PMMA domains. In effect, the homopolymer is sandwiched between the PMMA block brushes anchored to opposite sides of the domain.

When the homopolymer molecular weight is reduced to $12 \mathrm{~K}$, a very different picture of the distribution emerges, as seen in Figure $5 \mathrm{~b}$. In this case the homopolymer distribution is shown to be nearly uniform throughout the PMMA domain, with only a slight but detectable excess in the homopolymer concentration at the domain center. A much larger fraction of the short homopolymer chains is seen to penetrate the interfacial regions.

\section{Conclusions}

In the studies summarized above, neutron reflectivity gave quantitative information on chain distributions in ordered copolymer 
systems not accessible by traditional characterization methods. Results from selectively deuterated copolymers indicate that the junction points are highly localized to the copolymer interface while chain ends are distributed throughout the domain. In symmetric copolymer mixtures, the short copolymer component is seen to reside preferentially at the interface, while in copolymer-homopolymer blends, the homopolymer molecular weight strongly influences the chain distribution. For the first time, segment density profiles have been determined in such systems with sufficient detail to allow meaningful comparisons to theoretical models [10].
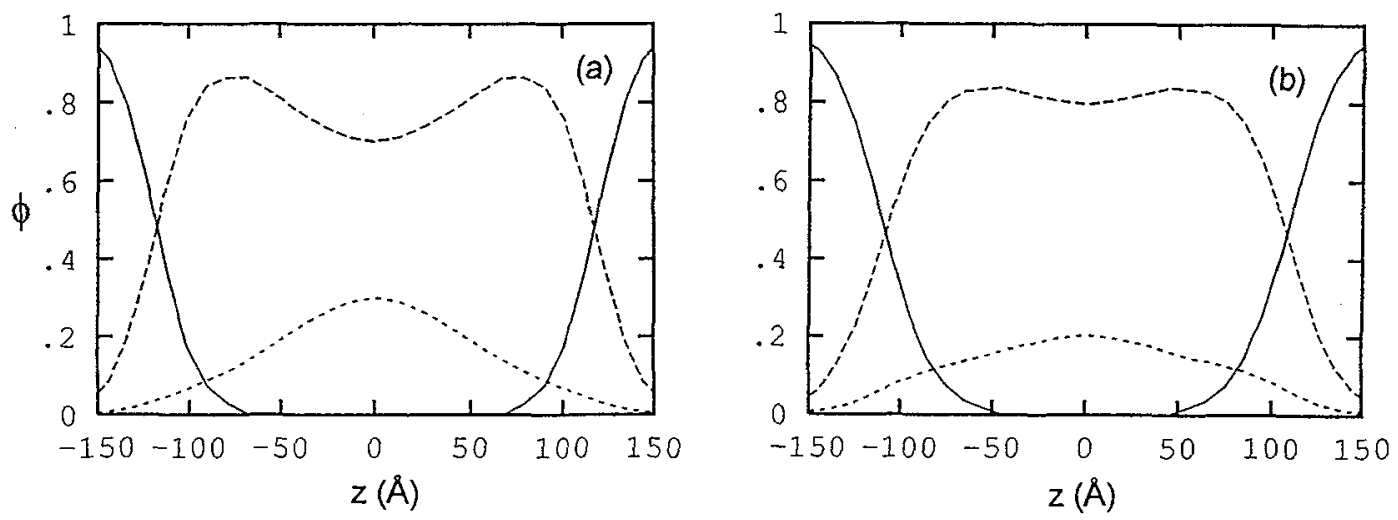

Figure 5. Distribution of long (a) and short (b) PMMA homopolymers in the PMMA domain of an ordered copolymer-homopolymer blend.

\section{References}

[1] Anastasiadis, S.H., Russell, T.P., Satija, S.K. and Majkrzak, C.F., J. Chem. Phys. 92 (1990) 5677.

[2] Foster, M.D., sikka, M., Singh, N., Bates, F.S., Satija, S.K. and Majkrzak, C.F., J. Chem. Phys. 96 (1992) 8605.

[3] Mayes, A.M. Johnson, R.D., Russell, T.P., Smith, S.D., Satija, S.K. and Majkrzak, C.F., Macromolecules 26 (1993) 1047.

[4] Mayes, A.M., Russell, T.P., Satija, S.K. and Majkrzak, C.F., unpublished.

[5] Mayes, A.M., Russeli, T.P., Satija, S.K. and Majkrzak, C.F., Macromolecules 25 (1992) 6531.

[6] Matsushida, Y., Mori, K., Saguchi, R., Noda, I., Nagasawa, M., Chang, T., Glinka, C.J. and Han, C.C., Macromolecules 23 (1990) 4317 .

[7] Hadziioannou, G. and skoulios, A., Macromolecules 15 (1982) 267.

[8] Hashimoto, T., Tanaka, H. and Hasegawa, H., Macromolecules 23 (1990) 4378 .

[9]' Winey, K.I., Thomas, E.L. and Fetters, L.J., Macromolecules 24 (1991) 6182.

[10] Shull,K.R., Mayes, A.M. and Russell, T.P., Macromolecules, in press. 